\title{
Ova Size and Endometrial Thickness Post-treatment with Vitamin D and Coenzyme Q10 Supplements in PCOs Women Resistant to Clomiphene
}

\section{Ansam Abdulameer Yahya ${ }^{1 *}$, Manal Khalid Abdulridha ${ }^{2}$ and HaiderAbass Al-Atar ${ }^{3}$}

${ }^{1}$ Babylon Health Department, Ministry of Health, Iraq

${ }^{2}$ Department of Clinical Pharmacy, College of Pharmacy, Mustansiriyah University,

Iraq

${ }^{3}$ Babylon Gynecology and Obstetrics Health Department, Ministry of Health, Iraq

*Corresponding Author: Ansam Abdulameer Yahya, Babylon Health Department, Ministry of Health, Iraq.

DOI: 10.31080/ASPS.2021.05.0809
Received: August 18, 2021

Published: October 23, 2021

(C) All rights are reserved by Ansam

Abdulameer Yahya., et al.

\begin{abstract}
Clomiphene Citrate resistance is a term that refers to the persistence of ovulation after standard Clomiphene Citrate therapy, and about $15 \%$ of Polycystic ovary syndrome were resistant to the maximum dose of clomiphene citrate. The study aims to evaluate the effect of combining oral vitamin D3 and CoQ10 supplements to overcome Clomiphene resistance on hormonal profile and ovulation outcome(ova size and endometrial thickness) in clomiphene citrate resistance PCOS patients. The study was a prospective intervention comparative, open-label study include 35 PCOS patient aged (18-34) years are resistant to clomiphene citrate ellocated in two groups, group 1 include vitamin D deficient PCOS patient received clomiphene citrate 100mg daily clomiphene for five days in each month with 10000IU daily vitamin D for 2 months, group 2 include PCOS patient with no vitamin D deficiency received $100 \mathrm{mg}$ daily clomiphene for five days in each month plus CoQ10 200mg daily for 2 months. Fasting blood samples used to measure serum estradiol, antimullerian hormone (AMH), luteinizing hormone (L.H.), and follicle-stimulating hormone (FSH) to calculate L.H.:FSH ratio, mid-cycle transvaginal ultrasound for determination of ova size and endometrial thickness. Both Vitamin D3 supplementation and CoQ10 supplementation resulted in a significant increase in ova size $(\mathrm{P}<0.001)$, while the increase in endometrial thickness was substantial only after vitamin $\mathrm{D}$ supplementation $(\mathrm{P}<0.001)$. Meanwhile, serum estradiol levels increased in both study groups after intervention but still not significant $(\mathrm{P}>0.05)$. In conclusion, receiving vitamin $\mathrm{D}$ and CoQ10 supplements by clomiphene resistance PCOS women improves serum hormone, including AMH and L.H.: FSH ratio, also improving ovulation outcome (ova size and endometrial thickness).
\end{abstract}

Keywords: Clomiphene Citrate Resistant; Polycystic Ovary Syndrome; Co-Q10; Vitamin D 3; Estradiol; Ovarian Functions; Endometrial Thickness

\section{Introduction}

On of the common endocrine disorder affect ibf women at reproductive age is the polycystic ovary syndrome (PCOS) is occur- ring in (4-12\%) of women, which affects reproductive performance and the overall health including the oligomenorrhea and obesity along with polycystic ovaries [1]. The oligomenorrhea, menstrual 
cycles last more than 35 days in eight cycles per year and consider as anovulatory processes; however, hyperandrogenemia with regular menstruation do not exclude chronic anovulation, especially in women with [2], since hirsutism considers the most common clinical sign of hyperandrogenemia and seen in approximately $60 \%$ of women with PCOS [3]. Clomiphene Citrate (CC) resistance, is a term which signifies persistence anovulation after standard CC therapy, in other words, failure to ovulate after receiving $150 \mathrm{mg}$ of CC daily for five days per cycle, for at least three cycles $[4,5]$. Clomiphene resistance occurs approximately in (15 to 40\%) of PCOS women and considers a significant challenge in gynecologic endocrinology 6 .

In PCOS women, estrogen secretion is characterized by chronic secretion without the cyclic pattern associated with the anovulatory cycle. Serum estradiol (E2) levels may vary in PCOS. Still, serum estrone $\left(E_{1}\right)$ levels are usually more significant than those of $E_{2}$ and this chronic estrogen secretion increase risk of endometrial hyperplasia and possibly the development of endometrial carcinoma [7]. A result of the lack of ovulation in PCOS leads to continuous high levels of estrogen. Insufficient progesterone results in increased serum luteinizing hormone (L.H.) levels and alterations in AntiMullerian Hormone (AMH) secretion. The higher the antral follicles count, the higher AMH levels, and women with PCOS typically have high antral follicles [8].

Vitamin D deficiency is common in PCOS, about) $67-85 \%$ (of PCOS women presented with 25-hydroxy Vitamin D (250HD) serum concentrations of $<20 \mathrm{ng} / \mathrm{ml}$ [9], vitamin D deficiency may strengthen symptoms of PCOS, in an observational studies lower 250HD levels potentiate insulin resistance, ovulatory and menstrual irregularities, lower pregnancy success rate, hirsutism, hyperandrogenism, obesity, and elevated cardiovascular disease risk factors [10], hence improved vitamin D status through supplementation can be theorized to hold potential for improving ovulation induction therapy, live birth rates, and reducing the risk of pregnancy loss in women with PCOS, a population that is already at enhanced risk for pregnancy wastage [11].

Coenzyme Q10 (CoQ10) is produced in mitochondria which is critical for the normal function of the body, especially reproduction, to facilitate energy production for the production of eggs, also; in women, oxidative stress cause mutations leading to decreased pregnancy rates and egg production, also increase abortion rate, accordingly, antioxidant effects of CoQ10 protects eggs from free radicals and oxidative stress; hence, CoQ10 supplementation may improved fertility [12,13]. Xu., et al. (2018) found that Pretreatment with CoQ10 improves embryological parameters in poor ovarian reserve in IVF-ICSI cycles [14]. Endogenous CoQ10 increases until 20 years of age, and then it decreases throughout life. Deficiency can be caused by biosynthesis impairment, insufficient feeding, or excessive body utilization [15].

They have taken together these facts. This study was designed to explore further the potential benefit of both supplements (Vitamin D3and CoQ10) in improving the quality of ova and endometrial thickness and their effectiveness in enhancing hormonal markers in PCOS women with clomiphene citrate resistance.

\section{Materials and Methods}

Patients

This prospective comparative open-label study of a total of 35 patients diagnosed CC resistant PCOS enrolled in the study. Patients ages of 18 and 40 and wanted to become pregnant, from private clinic's gynecologic and obstetric were enrolled. The patients were under the care of a gynecologist and were diagnosed using Rotterdam standards and treated according to practice guidelines. Resistant to clomiphene citrate (CC) monotherapy defined as persistent anovulation or ovulate with a very thin endometrium $<5$ $\mathrm{mm}$, on the day of HCG administration when the patient previously take induction therapy with clomiphene citrate alone for five days at least two or three cycles. Vitamin D deficient $(<20 \mathrm{ng} / \mathrm{ml})$ PCOS patients were enrolled in one group, and PCOS patients with no vitamin D deficiency enrolled in other group. The PCOS patients had oligomenorrhea for more than one month, progesterone (Allylestrenol) $5 \mathrm{mg}$ tablet twice daily for ten days used to attained menstrual bleeding, thereafter enrolled in the study. Patients with other gynecological and hormonal disorders are omitted from study.

The study received approval from the institution's scientific ethics committee and the general health directorate's approval. Patient informed permission was obtained after a thorough clarification of the study's intent, ensuring the accuracy of the data collected. This study is designed to evaluate combining oral vitamin D3 tablet or oral Co-enzyme Q10 with clomiphene citrate tablet on ovulation induction through ova size and endometrial thickness in 
CC-resistance PCOS patients, in addition to their effect on serum estradiol, L.H.:FSH ratio, and AMH. Group 1 patients included 21 patients treated with clomiphene citrate tablets 50mg twice daily after the meal for five days monthly with a 10000 IU daily dose of vitamin D3 oral tablets for two months. Group 2 include 14 patients received the same clomiphene citrate dosing plus $200 \mathrm{mg}$ daily dose of CO- Q10 capsule l for two months.

Transvaginal ultrasound at day ten and day 12of the cycle was performed to all patients to determine the number and size of the dominant follicle ( $\geq 18 \mathrm{~mm}$ ) in addition to endometrial thickness. When at least one strand measuring at least $(18 \mathrm{~mm})$ was detected, Pregnyl 5000-10,000 IU (HCG) injection was released. Patients were recommended to have sexual activity for the next 24-36 hours after receiving HCG injection. Patients' blood samples were taken after the two months for post-treatment assessment.

\section{Method}

Serum estradiol determination using ELISA estradiol kit by fully automated ELISA apparatus [16], serum L.H. and FSH determination using the MaglumiFSH (L.H.) assay, a sandwich chemiluminescence immunoassay $[17,18]$ then L.H.: FSH ratio calculation was done. The Ultra-Sensitive Antimullerian Hormone/Mullerian Inhibiting Substance (AMH/MIS) ELISA used to measure Serum Antimullerian Hormone (AMH) using sandwich immunoassay [19]. Transvaginal ultrasound, an examination of the female pelvis, was done for all patients in the study on days 10 and 12 of the cycle to follow up the ovulation (ova size and endometrial thickness) using an ultrasound machine radiologist [20]. And used for ova size and endometrial thickness determination to decide the time of human chorionic gonadotropin (hCG)administration when mature follicles are seen; that average endometrium thickness was $8 \mathrm{~mm}$ mature follicle size from 16-22 $\mathrm{mm}[21,22]$. The blood samples were first phenotyped for $\mathrm{ABO}$ blood groups (A, B, AB, and 0 ) using commercially available antisera (Biotest, Germany) utilizing a slide agglutination test [23].

\section{Statistical analysis}

The SPSS version 21 was used for categorical variables presented as number and percente. Continuous variables were presented as (Means \pm S.D.).The categorical variables were linked using the Fisher exact test. To equate the means of two classes, the indepen- dent samples t-test was used. For paired reading, the paired t-test was used to compare ways. Significant was described as a p-value of less than 0.05 .

\section{Results}

Socio-demographic and disease characteristics of PCOS patients on vitamin D3 and Co-Enzyme Q10 supplements

The patient demographic and Disease characteristics of $35 \mathrm{fe}-$ male patients are allocated as 21 patients in group 1 (58.5\%) and 14 patients in group 2 (41.5\%), Table (1). The mean age was 24.57 \pm 4 .16years for group 1 patients and $22.36 \pm 3.48$ years for group 2 patients. The body mass index for group 1 patients and group 2 patients was $27.16 \pm 4.66 \mathrm{~kg} / \mathrm{m} 2$ and $26.79 \pm 4.42 \mathrm{~kg} / \mathrm{m} 2$. Vitamin D level mean was $11.34 \pm 3.41$ and $36.68 \pm 17.75$ for patients in groups 1 and 2, respectively. Positive family history was recorded in (37.5\%) of patients in group 1 and $(29.4 \%)$ of patients in group 2. The disease history for patients in group 1 and 2 were as follows $19 \%$ versus $28.6 \%$ for less than 2 years duration, $71.4 \%$ versus $64.3 \%$ for $2-10$ years duration, and $9.6 \%$ versus $7.1 \%$ for more than 10years duration, there is no significant differences between study groups regarding age, BMI, family history and duration of symptom while a significant difference was seen between study groups regarding vitamin D level.

The ABO blood group phenotypes for patients in group 1 where patients carrying 0 phenotype represents (47.6\%), A phenotype patients (23.8\%), and the B and A.B. phenotypes means (14.3\%) for each phenotype, meanwhile, in group 2, higher percent (35.7\%) were A phenotype and (28.6\%) were 0 phenotypes while (21.4\%) and (14.3\%) of patients was B and A.B. phenotypes consequently, no significant difference between study groups regarding ABO blood group.

Among PCOS phenotype patients in group 1 and 2 were $66.7 \%$ versus $28.6 \%$ have all Rotterdam criteria (ovulatory dysfunction + PCOS on US+ androgen excess), and $23.8 \%$ versus $71.4 \%$ have (ovulatory dysfunction +PCOS on the U.S.) criteria, while (PCOS on U.S. + androgen excess) was seen only in two patients in group 1. No significant difference was found between both groups concerning all baseline socio-demographic and Disease characteristics data $(\mathrm{P}$ $>0.05$ ) except for Rotterdam criteria and vitamin D level that show a significant difference between both study groups $(\mathrm{P} \leq 0.05)$. 


\begin{tabular}{|c|c|c|c|}
\hline \multirow[b]{2}{*}{ Study variables } & \multicolumn{2}{|c|}{ Study groups } & \multirow[b]{2}{*}{ P-value } \\
\hline & $\begin{array}{l}\text { Group } 1 \\
(n=21)\end{array}$ & $\begin{array}{l}\text { Group } 2 \\
(n=14)\end{array}$ & \\
\hline \multirow[t]{2}{*}{ Age (years) } & $(24.57 \pm 4.16)$ & $(22.36 \pm 3.48)$ & $0 . .098^{\mathrm{NS}}$ \\
\hline & $\mathrm{n} \%$ & $\mathrm{n} \%$ & \\
\hline BMI $\left(\mathrm{kg} / \mathrm{m}^{2}\right)$ & $27.16 \pm 4.66$ & $26.79 \pm 4.42$ & $0.816^{\mathrm{NS}}$ \\
\hline Vitamin D level ( Baseline) & $11.34 \pm 3.41$ & $36.68 \pm 17.75$ & $<0.001^{*}$ \\
\hline \multicolumn{4}{|l|}{ Family history of PCOS } \\
\hline Yes & $9(42.9)$ & $4(28.6)$ & $0.488^{\mathrm{NS}} \mathrm{f}$ \\
\hline No & $12(57.1)$ & $10(71.4)$ & \\
\hline \multicolumn{4}{|l|}{ Duration of symptoms } \\
\hline$<2$ year & $4(19.0)$ & $4(28.6)$ & \\
\hline$(2-10)$ years & $15(71.4)$ & $9(64.3)$ & $0.800^{\mathrm{NS}}$ \\
\hline$>10$ years & $2(9.6)$ & $1(7.1)$ & \\
\hline \multicolumn{4}{|l|}{ Rotterdamcriteria } \\
\hline $\begin{array}{l}\text { Phenotype (A) Ovulatory dysfunction +PCOS on US+ androgen } \\
\text { excess }\end{array}$ & $\begin{array}{c}14(66.7) \\
0(0.0)\end{array}$ & $\begin{array}{l}4(28.6) \\
0(0.0)\end{array}$ & \\
\hline Phenotype (B) Ovulatory dysfunction +androgen excess & & & $0.017^{*}$ \\
\hline Phenotype (C) PCOS on US + androgen excess & $2(9.5)$ & $0(0.0)$ & \\
\hline Phenotype (D) Ovulatory dysfunction +PCOS on US & $5(23.8)$ & $10(71.4)$ & \\
\hline \multicolumn{4}{|l|}{ Blood group phenotypes } \\
\hline 0 & $10(47.6)$ & $4(28.6)$ & \\
\hline A & $5(23.8)$ & $5(35.7)$ & $0.699^{\text {NS }}$ \\
\hline $\mathrm{B}$ & $3(14.3)$ & $3(21.4)$ & \\
\hline$A B$ & $3(14.3)$ & $2(14.3)$ & \\
\hline
\end{tabular}

Table 1: Baseline socio-demographic and Disease characteristics of patients.

Data presented as mean \pm S.D., Number (n), Percentage (\%), No significant differences (NS) (P > 0.05), *: significant difference (P $\leq 0.05)$,

f: Fisher exact test used for statistical analysis of (family history).

Independent -sample t-test is used for statistical analysis of (age, BMI, vitamin D).

Chi-square test is used for statistical analysis of (blood group, Rotterdam criteria, duration of symptoms).

Effect of vitamin D3 and co-enzyme Q10 supplements on serum estradiol level (E2), antimullerian hormone ( $\mathrm{AMH})$, and

\section{L.H.:FSH ratio}

In the pretreatment mean estradiol level, no significant difference was noticedbetween group 1 and group 2 patients $(\mathrm{P}>0.05)$, post-treatment increase in estradiol in both study groups was nonsignificant when compared to pretreatment level ( $P>0.05$ ), also no significant difference between both study groups after treatment $(\mathrm{P}>0.05)$. In contrast, the pretreatment means AMH shows 
a significant difference between group 1 and group 2 patients $(\mathrm{P} \leq$ 0.05), and post-treatment, the difference was non-significant. Still, both study group post-treatment improvement was significant $(\mathrm{P}$ $\leq 0.05$ ), L.H.: FSH ratio means no significant difference was noticed between group 1 and group 2 pre and post-treatment. In contrast, the decrease in the ratio in both study group after the intervention was significant $(\mathrm{P} \leq 0.05)$, (Table 2$)$.

\begin{tabular}{|l|c|c|c|}
\hline \multirow{2}{*}{ Study variable } & \multicolumn{2}{|c|}{ Study group } & \multirow{2}{*}{ P-value } \\
\cline { 2 - 3 } & Group 1 & Group 2 & \\
\hline E2 $(\mathrm{pg} / \mathrm{ml})$ & $\mathrm{n}=21$ & $\mathrm{n}=14$ & \\
\hline Pre treatment & $89.13 \pm 41.43$ & $81.91 \pm 21.40$ & $0.36^{\mathrm{NS}}$ \\
\hline Post treatment & $122.54 \pm 88.83$ & $83.95 \pm 25.34$ & $0.072^{\mathrm{NS}}$ \\
\hline P-value & $0.122^{\mathrm{NS}}$ & $0.826^{\mathrm{NS}}$ & \\
\hline $\begin{array}{l}\text { Anti-mullerian } \\
\text { hormone AMH } \\
\text { (ng/ml) }\end{array}$ & & & \\
\hline Pre treatment & $4.75 \pm 2.76$ & $4.08 \pm 3.10$ & $0.020^{*}$ \\
\hline Post treatment & $3.51 \pm 1.56$ & $2.96 \pm 1.88$ & $0.067^{\mathrm{NS}}$ \\
\hline P-value & $0.003^{*}$ & $0.022^{*}$ & \\
\hline LH/FSH ratio & & & \\
\hline Pre treatment & $1.11 \pm 0.40$ & $1.35 \pm 0.55$ & $0.185^{\mathrm{NS}}$ \\
\hline Post treatment & $0.86 \pm 0.31$ & $0.80 \pm 0.24$ & $0.519^{\mathrm{NS}}$ \\
\hline P-value & $0.019^{*}$ & $0.001^{*}$ & \\
\hline
\end{tabular}

Table 2: Effect of Vitamin D3 and Co-Enzyme Q10 Supplements on Serum Hormonal Level.

Data presented as mean \pm S.D., Number of patients (n).

NS: No significant differences $(\mathrm{P}>0.05)$, *: significant difference

$$
(\mathrm{P} \leq 0.05) \text {. }
$$

Effect of vitamin D3 and co-enzyme Q10 supplements on ova size and endometrial thickness (E.T.)

The mean ova size of patients in group 1 and group 2 showed no significant difference Pretreatment ( $\mathrm{P}>0.05$ ); nevertheless, a significant increase in the mean ova size in both groups 1and group 2 was produced post-treatment with both supplements when compared to pretreatment level $(\mathrm{P}<0.05)$ after two months of intervention, (Table 3). Moreover, the mean endometrial thickness in patients between group 1 and group 2 was not different pretreatment ( $\mathrm{P}>0.05)$; however, a significant increase in endometrial thickness was revealed in group 1 patients when compared to pretreatment level $(\mathrm{P}<0.05)$ after two months of intervention, meanwhile the increase was non-significant in group 2 patients after intervention $(\mathrm{P}>0.05)$.

\begin{tabular}{|l|c|c|c|}
\hline \multirow{2}{*}{ Study variable } & \multicolumn{2}{|c|}{ Study group } & \multirow{2}{*}{ P-value } \\
\cline { 2 - 3 } & Group 1 & Group 2 & \\
\hline Ova size (mm) & $\mathrm{n}=21$ & $\mathrm{n}=14$ & \\
\hline Pre treatment & $11.06 \pm 4.9$ & $12.66 \pm 4.82$ & $0.311^{\mathrm{NS}}$ \\
\hline Post treatment & $17.15 \pm 4.12$ & $19.18 \pm 3.87$ & $0.155^{\mathrm{NS}}$ \\
\hline P-value & $<0.001^{*}$ & $0.001^{*}$ & \\
\hline $\begin{array}{l}\text { Endometrial } \\
\text { thickness (mm) }\end{array}$ & & & \\
\hline Pre treatment & $6.76 \pm 1.48$ & $7.48 \pm 1.74$ & $0.214^{\mathrm{NS}}$ \\
\hline Post treatment & $8.36 \pm 1.66$ & $7.86 \pm 2.27$ & $0.488^{\mathrm{NS}}$ \\
\hline P-value & $<0.001^{*}$ & $0.526^{\mathrm{NS}}$ & \\
\hline
\end{tabular}

Table 3: Effect of vitamin D3 and coenzyme Q10 supplements on Ova size and Endometrial Thickness.

Data presented as mean \pm S.D., Number of patients (n).

NS: No significant differences $(\mathrm{P}>0.05),{ }^{*}(\mathrm{P} \leq 0.05)$ is considered a significant difference.

\section{Discussion}

Many of the PCOS patients in this sample were resistant to clomiphene citrate and wanted to get pregnant, and they were between the ages of 18 and 34, as in the majority of prior trials [24-27]. Most PCOS patients in the current study were overweight and obese. This finding was consistent with that of previous studies where more than half of enrolled patients were obese [28,29], weight gain occur in (61\%) and (76\%) of PCOS women [30]. Hyperinsulinemia, insulin resistance, and hyperandrogenemia affect adipocyte activity and distribution by inhibiting adipocyte differentiation, which regulates lipolysis and lipogenesis [31].

Positive family history of PCOS in the present study was between (30\%-40\%) of patients. Similarly (45\% - 60.8\%) of PCOS patients have a positive family history as reported previously [32], and this is due to the fact of a hereditary component of PCOS and family 
association of reproductive and metabolic abnormalities causing increased risk of among first-degree relatives of PCOS women [33]. Another study found a 5 - 6 fold increase among first-degree relatives compared with PCOS prevalence in the general population [34]. In this study, most PCOS patients enrolled have duration of symptoms of a (2-10) years which is the leading cause of CC resistance or increase symptoms.

PCOS phenotype distribution in this study population fulfilled two out of three Rotterdam criteria giving a PCOS phenotype A [ovulatory dysfunction+polycystic ovary on US+androgen excess] reported in 18 women (14 of them in group 1 and 4 of them in group 2), PCOS phenotype B [ovulatory dysfunction and clinical and/or biochemical hyperandrogenism] was not observed in the present study patients, PCOS phenotype C [polycystic ovary on the U.S. and clinical and/or biochemical hyperandrogenism] only presented in 2 women in group 1, PCOS phenotype D [ovulatory dysfunction and polycystic ovary on the U.S.] repotted in 15 women (5 of them in group 1 and 10 of them in group 2).This finding showed that a higher percentage of enrolled patients carry PCOSphenotype A; this was inconsistent with previous studies [35,36]; meanwhile, other studies showed a higher percent of phenotype C (37-39\%), as different phenotypes were less prevalence [37-40]. Most PCO women enrolled carry blood group 0 group phenotype, the finding in line with that of previous studies [41-43].

Estrogen has the potential to directly scavenge free radicals, enhance the function of certain naturally occurring antioxidant enzymes (particularly glutathione peroxidase), and function as a chain-breaking antioxidant in vivo when present in high concentrations [44]. On the other hand, anovulatory women have a comparatively steady-state estradiol level. They frequently have oligo- or amenorrhea since estrogen levels peak twice in the cycle, once in the late follicular phase, and to a lesser extent in the luteal phase [45]. Serum estradiol was tested during the early follicular phase in this study, and the baseline was varied but still within the normal range for serum estradiol during the follicular phase. After the intervention, serum estradiol increased in both classes, particularly after vitamin D supplementation. There was no previous research to interpret the impact of vitamin D or CoQ10 on serum estradiol levels. Still, the ambiguous effect could be due to the time of testing estradiol and the limited sample size of patients after excluding the patients who became pregnant in the study, since the risk of extreme elevation of serum estradiol during pregnancy, which was observed in the current study, could be due to the time of testing estradiol and the small sample size of patients after excluding the patients who became pregnant in the [46].

In PCOS women, an alteration in gonadotropin-releasing hormone secretion, causing an increase in luteinizing hormone (L.H.) secretion with normal follicle-stimulating hormone (FSH) secretion, has been observed and widely accepted as specific endocrine profiles [47], this profile gives rise to an abnormal LH/FSH ratio in many patients, and a valuable diagnosis biomarker of PCOS [48]. Hence, the L.H.: FSH ratio of (1: 2) when presented ( $<1)$ it produce good ovulation and more mature follicle, according to the Wiser., et al. whom reported that the pregnancy rate in women with L.H.: FSH ratio of ( $>1.5$ ) was significantly lower $(16.7 \%)$ than in those with a balance of (0.5-1.5) (40.4\%), despite the nearly normal levels of both hormones [49]. In the present study, the L.H.: FSH ratio was significantly reduced $(\mathrm{P}<0.05)$ after both vitamin D3 and CoQ10 therapy compared to the pretreatment level. However, the effect of alfacalcidol alone and combined with metformin in PCOS women produced no significant change in LH/FSH ratio after six months of therapy in the previous study [50].

Excessive ovarian development of anti-mullerian hormone $(\mathrm{AMH})$, secreted by an excessive amount of developing follicles, has long been thought to be a key feature of PCOS, with growing data in the last decade supporting AMH's function in the syndrome's pathogenesis [48]. The baseline AMH was elevated in both groups, with a higher value in the hypovitaminosis PCOS patients. After two months of vitamin D3 and Co Q10 supplementation, the level significantly decreased to a near-normal level. Irani., et al. found that giving 50000IU vitamin D3 to PCOS women deficient in vitamin D once a week for eight weeks reduced and normalized AMH levels relative to placebo, resulting in better fertility outcomes [51]. Moreover, The results mirrored the existing findings, with mean values changing from $4.882 \mathrm{ng} / \mathrm{ml}$ to $3.792 \mathrm{ng} / \mathrm{ml}$. Following the action, the proportion of women with stabilized AMH levels (4 ng/ $\mathrm{ml}$ ) increased from $20 \%$ to $80 \%$, indicating that endogenous vitamin D levels have a critical regularizing impact on ovarian reserves in PCOS patients with vitamin D deficiency [52]. Despite the lack of previous studies to interpret the effect of CoQ10 supplementation on AMH levels in PCOS patients, one of the possible mechanisms of 
CoQ10 in clomiphene-citrate-resistant PCOS patients involves cellular ATP formation.

Reduces oxidative stress in the ovary, which reduces the synthesis of pro-inflammatory cytokines and maintains plasma membrane integrity. Tissue depletion of CoQ10, a micronutrient, or an antiapoptotic pathway implicated in follicular cohort atresia may indirectly affect AMH levels.

Ova size in the present study was increased significantly after both interventions, and this improvement probably due to decreasing oxidative stress after use of antioxidants such as vitamin D and CoQ10 supplementations, and since oxidative stress considered as a critical signal in the initiation of apoptosis in antral follicles and granulosa cells of antral hairs by diverse stimuli, such as exposure to radiation, exogenous toxicants, or gonadotropin withdrawal, so that antioxidants protect against these stimuli [53]. This improvement in ova size in the present study led to improved overall ovulation stimulation as reported in the previous study where the overall ovulation stimulation was in $75 \%$ of PCOS patients; meanwhile, comprehensive pregnancy was $15 \%$ equal in both groups [54]. No previous studies had explored the effect of vitamin D or CoQ10 on ova size to the best search.

Finally, the endometrial thickness in the present study was increased significantly after vitamin D supplementation, as in agreement with studies by Rasekhjahromi Athar., et al. and Asadi., et al. $[55,56]$; meanwhile, no effect of CoQ10 on endometrial thickness was observed. Controversy data was reported previously regarding the effect of CoQ10 on endometrial thickness, Lakshmi., et al.'s finding stated that endometrial thickness in clomiphene citrate plus CoQ10 was significantly lowered than in clomiphene citrate only group [25]. Conversely, the study by Refaeey., et al. revealed that endometrial thickness considerably higher in the clomiphene citrate plus CoQ10 group than in the clomiphene citrate only group [24]. Nevertheless, following the overall results of the present study, clinical pregnancy occurs equally in 3 PCOS patients after receiving vitamin D and Co Q10 supplements in each study group.

\section{Conclusion}

Based on the findings of this report, oral vitamin D and CoQ10 supplementation improves reproductive outcomes while also improving hormonal profiles in clomiphene citrate resistance PCOS patients (ova size and endometrial thickness); a more extensive study is needed to see how vitamin D3 and CoQ10 supplementation affects metabolic status and other hormonal profiles including SHBG, TSH, and prolactin in PCOS patients, particularly after vitamin D status is restored.

\section{Acknowledgment}

The authors would like to thank all participants for providing the practice platform of this study.

\section{Conflict of Interest}

The authors report no conflicts of interest in this work.

\section{Bibliography}

1. El Hayek S., et al. "Poly Cystic Ovarian Syndrome: An Updated Overview". Frontiers in Physiology 7 (2016): 124.

2. Azziz R., et al. "Task Force on the Phenotype of the Polycystic Ovary Syndrome of The Androgen Excess PCOS Society. The Androgen Excess and PCOS Society criteria for the polycystic ovary syndrome: the complete task force report". Fertility Sterility 91 (2009): 456-488.

3. R Dumitrescu., et al. "The Polycystic Ovary Syndrome: An update on metabolic and hormonal mechanisms". Journal of Medicine and Life 8.2 (2015): 142-145.

4. Brown J., et al. "Clomiphene and anti-oestrogens for ovulation induction in PCOS". Cochrane Database of Systematic Reviews 4 (2009).

5. National Collaborating Centre for Women's and Children's Health/National Institue for Clinical Excellence. Fertility: assessment and treatment for people with fertility problems. Clinical Guideline No. 11, RCOG Press, London, UK (2004).

6. HASHIM, Hatem Abu. Management of Women with Clomifene Citrate Resistant Polycystic Ovary Syndrome-An EvidenceBased Approach. In: Polycystic Ovary Syndrome. InTech (2012).

7. Chang R and Kazer R. Glob. Lib. women's med (2014).

8. Tola H., et al. "Assessment of the Role of the Anti-Mullerian Hormone, Luteinizing Hormone/Follicle Stimulating Hormone Ratio in the Diagnosis of Polycystic Ovary Syndrome in 
Sudanese Women". Open Access Macedonian Journal of Medical Sciences 6.7 (2018): 1244-1247.

9. Thomson RL., et al. "Vitamin D in the etiology and management of polycystic ovary syndrome". Clinical Endocrinology 77.3 (2012): 343-350.

10. Dipanshu Sur and R Chakravorty. "The relationship between vitamin D, insulin resistance, and infertility in PCOS women". Gynecology and Obstetrics (Sunnyvale) 5.294 (2015): 21610932.1000294.

11. Pal L., et al. "Vitamin D Status Relates to Reproductive Outcome in Women With Polycystic Ovary Syndrome: Secondary Analysis of a Multicenter Randomized Controlled Trial”. The Journal of Clinical Endocrinology and Metabolism 101.8 (2016): 30273035.

12. Daniel Levine MD. CoQ10, and Fertility (2019).

13. Giannubilo SR., et al. "CoQ10 Supplementation in Patients Undergoing IVF-ET: The Relationship with Follicular Fluid Content and Oocyte Maturity". Antioxidants (Basel) 7.10 (2018): 141.

14. Xu., et al. "Pretreatment with coenzyme Q10 improve ovarian response and embryo quality in low-prognosis young women with decreased ovarian reserve: a randomized controlled trial". Reproductive Biology and Endocrinology 16 (2018): 29.

15. Gouveia FL. "Coenzyme Q10 and polycystic ovary syndrome: Systematic review". Clinical and Medical Investigations 3.4 (2018): 1-4.

16. Human serum Estradiol ELISA kit (catalog No: 4925-300) [product insert on the internet]. USA: Mobobind.

17. Human serum FSH Maglumi kit (catalog No: 130202001M) China: Snipe.

18. Human serum LH Maglumi kit (catalog No: 130202002M) [product insert on the internet]. China: Snibe.

19. Human serum AMH ELISA kit (catalog No: 032417) [product insert on the internet]. USA: AnshLabs.

20. Hoffman B. Polycystic Ovarian Syndrome and Hyperandrogenism, in Williams GYNECOLOGY. 3rd ed. New York: McGraw-Hill
Education (2016): 387-389-397.

21. Panchal S and Nagori CB. "Follicular Monitoring". Donald School Journal of Ultrasound in Obstetrics and Gynecology 6.3 (2012): 300-312.

22. Ochie Kalu., et al. "Sonographic Assessment Of The Foliclecular Size And Endometrial Thickness In Apparently Normal Women And Among Women With Infertility". International Journal of Advanced Research 3.5 (2015): 1223-1228.

23. Eckhardt M. "Blood Groups Serology". A. G. Medizinische Information. W. Germany (1975): 12-13.

24. El Refaeey A., et al. "Combined coenzyme Q10 and clomiphene citrate for ovulation induction in clomiphene-citrate-resistant polycystic ovary syndrome". Reproductive Biomedicine Online 29.1 (2014): 119-124.

25. Lakshmi B., et al. "A study on quality of life and effect of coenzyme-Q10 in polycystic ovarian syndrome patients". International Journal of Advances in Pharmacy Medicine and Bioallied Sciences 6.1 (2018): 14-21.

26. Almoayad HA., et al. "Comparison of antimullerian hormone level between women with polycystic ovary syndrome and normal ovulatory infertile women of reproductive age". Iraqi Journal of Medical Sciences 15.3 (2017): 234-241.

27. Maktabi M., et al. "The effects of vitamin D supplementation on metabolic status of patients with polycystic ovary syndrome: a randomized, double-blind, placebo-controlled trial". Hormone and Metabolic Research 49.7 (2017): 493-498.

28. Wang F., et al. "Analyses of optimal body mass index for infertile patients with polycystic or non-polycystic ovary syndrome during assisted reproductive treatment in China". Scientific Reports 6 (2016): 34538.

29. Gomathi K., et al. "Biochemical parameters in women with polycystic ovary syndrome in Ajman, UAE". Nepal Journal of Obstetrics and Gynaecology 6.2 (2012): 7-10.

30. El Hayek S., et al. "Poly Cystic Ovarian Syndrome: An Updated Overview". Frontiers in Physiology 7.124 (2016).

Citation: Ansam Abdulameer Yahya., et al. "Ova Size and Endometrial Thickness Post-treatment with Vitamin D and Coenzyme Q10 Supplements in PCOs Women Resistant to Clomiphene". Acta Scientific Pharmaceutical Sciences 5.11 (2021): 45-54. 
31. Vilmann LS., et al. "Development of obesity and polycystic ovary syndrome in adolescents". Hormone Research in Pediatrics 78.5-6 (2013): 269-278.

32. Tehrani HG., et al. "The effect of calcium and vitamin D supplementation on the menstrual cycle, body mass index and hyperandrogenism state of women with the polycystic ovarian syndrome". Journal of Research in Medical Sciences: The Official Journal of Isfahan University of Medical Sciences 19.9 (2014): 875 .

33. Begum GS., et al. "Assessment of Risk Factors for development of Polycystic Ovarian Syndrome". Diabetes 1 (2017): 2.

34. Kausar-Miller MD., et al. "Prevalence of polycystic ovary syndrome (PCOS) in first-degree relatives of patients with PCOS". Fertility and Sterility 75.1 (2001): 53-58.

35. Ma YM., et al. "Characteristics of abnormal menstrual cycle and polycystic ovary syndrome in community and hospital populations". Chinese Medical Journal (Engl) 123 (2010): 2185-2189.

36. Moran C., et al. "Prevalence of polycysticovary syndrome and related disorders in Mexican women". Gynecologic and Obstetric Investigation 69 (2010): 274-280.

37. Tehrani FR., et al. "The Prevalence of metabolic disorders in various phenotypes of polycystic ovary syndrome: a community-based study in Southwest of Iran". Reproductive Biology and Endocrinology 12 (2014): 89.

38. Lauritsen MP., et al. "The Prevalence of polycystic ovary syndrome in an average population the Rotterdam criteria versus revised criteria including anti-Mullerian hormone". Human Reproduction 29 (2014): 791-801.

39. Li R., et al. "Prevalence of polycystic ovary syndrome in women in China: a large community-based study". Human Reproduction 28 (2013): 2562-2569.

40. Yildiz BO., et al. "Prevalence, phenotype and cardiometabolic risk of polycystic ovary syndrome under different diagnostic criteria”. Human Reproduction 27 (2012): 3067-3073.

41. Aaiad Hameed Aajil. "The Association Between HLA-Class I Antigens and Polycystic Ovary Syndrome in a Sample of Iraqi
Patients". American Journal of Medical Genetics 4.1 (2011): 5256.

42. Chatterjee., et al. "Epidemiological correlate among women with Polycystic ovary syndrome in South India". International Journal of Current Research and Academic Review 2.9 (2014): 181-186.

43. Rahul P., et al. "Polycystic Ovary Syndrome, Blood Group and Diet: A Correlative Study In South Indian Females". International Journal of Medical Research and Health Sciences (IJMRHS) 3.3 (2014): 604-609.

44. Dana M., et al. "Impact of Serum Estradiol on Biomarkers of Oxidative Stress in Polycystic Ovary Syndrome and Ovulatory Women". Clinical Medicine Insights: Women's Health 5 (2012): 45-51.

45. Speroff L and Fritz MA. "Anovulation and the polycystic ovary". Clinical Gynecologic Endocrinology and Infertility. 7th ed. Philadelphia, PA: Lippincott, Williams and Wilkins (2015): 465-498.

46. KUIPER E A M., et al. "Reproductive hormone concentrations in pregnancy and neonates: a systematic review". Reproductive Biomedicine Online 27.1 (2013): 33-63.

47. Normal RJ., et al. "Polycystic ovary syndrome". Lancet 370 (2007): 685-697.

48. LE Minh Tam., et al. "Explored the role of anti-Mullerian hormone and LH/FSH ratio in the diagnosis of polycystic ovary syndrome". Clinical Endocrinology 90.4 (2019): 579-585.

49. Wiser A., et al. "Effect of high LH/FSH ratio on women with polycystic ovarysyndrome undergoing in vitro maturation treatment". The Journal of Reproductive Medicine 58.5-6 (2013): 219-223.

50. Dravecká I., et al. "The effect of alfacalcidol and metformin on phenotype manifestations in women with polycystic ovary syndrome-a preliminary study". Physiological Research 65.5 (2016): 815.

51. Irani M., et al. "Vitamin D supplementation appears to normalize serum AMH levels in vitamin D deficient premenopausal women". Fertility and Sterility 100.3 (2013): S338. 
52. S Sharma. "Evaluation of the effect of vitamin D supplementation onserum AMH in vitamin D deficient PCOS women" (2018).

53. Devine P J., et al. "Roles of reactive oxygen species and antioxidants in ovarian toxicity". Biology of Reproduction 86.2 (2011):

27.

54. Ansam Abdulameer Yahya., et al. "The Effect of Vitamin D and Co-enzyme Q10 Replacement Therapy on Hormonal Profile and Ovulation Status in Women with Clomiphene Citrate Resistant Polycystic Ovary Syndrome". Journal of Pharmaceutical Sciences and Research 11.1 (2019): 208-215.

55. Rasekhjahromi Athar., et al. "The effect of vitamin D in the treatment of infertile PCOS patients".

56. Asadi M., et al. "Vitamin D improves endometrial thickness in PCOS women who need intrauterine insemination: a randomized, double-blind placebo-controlled trial". Archives of Gynecology and Obstetrics 289.4 (2013): 865-870.,

\section{Volume 5 Issue 11 November 2021}

(c) All rights are reserved by Ansam Abdulameer Yahya., et al. 